\title{
A Syntactic Characterization of the Gabbay-de Jongh Logics
}

\author{
Jeroen P. Goudsmit \\ Utrecht University, Utrecht, The Netherlands \\ J.P.Goudsmit@uu.nl
}

\begin{abstract}
Skura [12] syntactically characterised intuitionistic propositional logic among all intermediate logics by means of a Łukasiewicz-style refutation system. Another such syntactic characterisation is given by Iemhoff $[5]$ in terms of admissible rules. Here we offer a bridge between these results. That is to say, we provide sufficient conditions under which admissible rules yield a refutation system fully characterising the logic. In particular, we give a characterisation of the Gabbay-de Jongh logics by means of refutation systems employing ideas from admissibility.

Lukasiewicz [9] introduced refutation systems as a formal means of reasoning about derivability and non-derivability within the same system. A refutation system inductively defines non-derivability, so in a very real way a refutation system is a proof system for non-provability. Proofs in this system are called "refutations", and when a refutation can be built for a formula $\phi$ we call it refutable, denoted by $\dashv \phi$. A refutation system is said to be sound (with respect to a certain logic) if all refutable formulae are non-derivable and we call it complete when the converse holds. Whenever the logic at hand comes with semantics and a sound refutation system one can read $\dashv \phi$ as "there exists a counter model of $\phi$ ".

The original refutation system given by Łukasiewicz was intended for classical propositional logic (CPC). It consisted of the rules Ax, Subs and MT (modus tollens) as shown below, where $\vdash$ is to be read as derivability within CPC. Bearing in mind that CPC is complete with respect to Boolean algebras one can readily derive both soundness and completeness of the system. Łukasiewicz [10] proposed another refutation system for intuitionistic propositional logic (IPC), which consisted of the rules listed below. Naturally, here $\vdash$ is to be read as derivability within IPC. In stating that this system is a complete refutation system for IPC he, in essence, conjectured that IPC is the sole intermediate logic with the disjunction property. The situation turned out to be more subtle, for there are continuum many such intermediate logics [13].

$$
\frac{-\mathrm{Ax}}{\dashv p} \quad \frac{\dashv \sigma(\phi)}{\dashv \phi} \text { Subs } \quad \frac{\dashv \psi \quad \phi \vdash \psi}{\dashv \phi} \mathrm{MT} \quad \frac{\dashv \phi \quad \vdash \psi}{\dashv \phi \vee \psi} \mathrm{DP}
$$

The rule DP is basically the counter-positive of the disjunction property, which states that if $\phi \vee \psi$ is derivable, then one amongst $\phi$ and $\psi$ must be derivable. Stronger versions of this rule are actually valid for IPC. It was mentioned by Kreisel and Putnam 8 and proven by Harrop [4 that derivability within IPC of $\neg \chi \rightarrow \phi \vee \psi$ entails the derivability of one amongst $\vdash \neg \chi \rightarrow \phi$ and $\vdash \neg \chi \rightarrow \phi$. Skura [12] generalised these rules even further and proved that replacing DP by the refutation rules corresponding to these generalised disjunction properties suffices to refute all non-theorems of IPC. This result gives a syntactic characterisation of IPC amongst all intermediate logics.

The generalised disjunction rules show great similarity to the Visser rules, a well-studied scheme of rules admissible in IPC [7]. Iemhoff [5] in fact characterised IPC as the sole intermediate logic that admits them.
\end{abstract}


Gabbay and de Jongh 2 introduced an infinite series of logics stratified over the natural numbers, where the $n^{\text {th }}$ logic in this sequence is complete with respect to finite trees with at most $(n+1)$-fold branching. As such it is equal to $\mathrm{BB}_{n}$ [1]. It has been shown [3 that a restricted version of the Visser rules suffices to axiomatise admissibility over the Gabbay-de Jongh logics. We give a characterisation of the $\operatorname{logics} \mathrm{BB}_{n}$ for all $n \neq 1$ by a restricted form of Skura's refutation system for IPC.

Let us get a bit more technical. Consider any intermediate logic, that is to say, a consistent axiomatic extension of IPC, and let $\vdash$ denote derivability within this logic. A rule is a pair, written $\Gamma / \Delta$ where $\Gamma$ and $\Delta$ are both finite sets of formulae. Such a rule is said to be admissible, denoted $\Gamma m \Delta$, when for all substitutions $\sigma$ one has that

$$
\text { if } \vdash \sigma(\phi) \text { for all } \phi \in \Gamma \text { then } \vdash \sigma(\chi) \text { for some } \chi \in \Delta \text {. }
$$

Note that all derivable rules are admissible, that is to say, if $\Gamma \vdash \chi$ then $\Gamma m \chi$. The following are examples of rules that can be admissible. The left-most rule is the disjunction property and the right-most rules are known as the (strong) Visser rules. Note that each rule is a generalisation of the former, although by [1] the second is admissible precisely if the first is.

$$
\frac{\phi \vee \psi}{\{\phi, \psi\}} \quad \frac{\neg \chi \rightarrow \phi \vee \psi}{\{\neg \chi \rightarrow \phi, \neg \chi \rightarrow \psi\}} \quad \frac{\left(\bigwedge_{i=1}^{n} \phi_{i} \rightarrow \psi_{i}\right) \rightarrow \bigvee_{i=n+1}^{n+m} \phi_{i}}{\left\{\left(\bigwedge_{i=1}^{n} \phi_{i} \rightarrow \psi_{i}\right) \rightarrow \phi_{j} \mid j=1, \ldots, n+m\right\}}
$$

If $\chi$ is classically non-derivable it follows that $\forall \chi$. Consequently, if $\phi$ m $\Delta$ for some set of CPC-non-derivable formulae $\Delta$, then $\forall \phi$ follows. These observations motivate the following theorem connecting refutation systems and admissibility. Whenever the prerequisites of the theorem are met, the intermediate logic at hand has a sound and complete refutation system. As a refutation system can only be sound and complete for one unique intermediate logic this gives a characterisation of the logic. When we can moreover restrict the amount of rules to a syntactically describable set of rules, this characterisation can in all fairness be called syntactic.

Theorem 1. Suppose that for all formulae $\phi$ one has $\forall \phi$ if and only if there is a set of CPCnon-derivable formulae $\Delta$ and a substitution $\sigma$ such that $\sigma(\phi) m \Delta$. The predicate $\dashv$ inductively defined as below now satisfies $\forall=\dashv$.

$$
\frac{\dashv \sigma(\phi)}{\dashv \phi} \quad \frac{\dashv \psi \quad \phi \vdash \psi}{\dashv \phi} \quad \frac{\dashv \chi \text { for all } \chi \in \Delta \quad \phi m \Delta}{\dashv \phi}
$$

Note that CPC trivially satisfies the property supposed by the above theorem. We can prove that all the Gabbay-de Jongh logics satisfy it as well. As an immediate consequence we thus obtain a complete refutation system for this infinite chain of intermediate logics. The result can furthermore be strengthened by restricting the amount of rules included in the inductive definition of the predicate $\dashv(-)$.

Iemhoff [6] proved that all admissible rules of IPC follow from the Visser rules, and this result nicely stratifies over the Gabbay-de Jongh logics [3. This suggests that in the above definition of $\dashv(-)$ it suffices to restrict the right-most clause to those rules arising from Visser rules. This turns out to be the case, culminating in the following theorem which gives a syntactic characterisation of the Gabbay-de Jongh logics.

Theorem 2. Let $n \in \mathbb{N}$ be such that $n \neq 1$. The refutation determined by the rules $\mathrm{Ax}$, Subs, $\mathrm{MT}$ and $\mathrm{GDP}_{n}$ is sound and complete for $\mathrm{BB}_{n}$. 


\section{References}

[1] Alexander Chagrov and Michael Zakharyaschev. Modal Logic, volume 77 of Oxford Logic Guides. Oxford University Press, 1997.

[2] Dov M. Gabbay and Dick H.J. de Jongh. A Sequence of Decidable Finitely Axiomatizable Intermediate Logics with the Disjunction Property. The Journal of Symbolic Logic, 39(1):67-78, 1974.

[3] Jeroen P. Goudsmit and Rosalie Iemhoff. On unification and admissible rules in Gabbay-de Jongh logics. Logic Group Preprint Series, 297:1-18, 2012.

[4] Ronald Harrop. Concerning Formulas of the Types $a \rightarrow a \vee b, a \rightarrow(\exists x) b(x)$ in Intuitionistic Formal Systems. The Journal of Symbolic Logic, 25(1):27-32, 1960.

[5] Rosalie Iemhoff. A(nother) characterization of intuitionistic propositional logic. Annals of Pure and Applied Logic, 113(1-3):161-173, 2001. First St. Petersburg Conference on Days of Logic and Computability.

[6] Rosalie Iemhoff. On the Admissible Rules of Intuitionistic Propositional Logic. The Journal of Symbolic Logic, 66(1):281-294, 2001.

[7] Rosalie Iemhoff. Intermediate Logics and Visser's Rules. Notre Dame Journal of Formal Logic, 46(1):65-81, 2005.

[8] Georg Kreisel and Hilary Whitehall Putnam. Eine Unableitbarkeitsbeweismethode für den Intuitionistischen Aussagenkalkül. Archiv für mathematische Logik und Grundlagenforschung, 3:74-78, 1957.

[9] Jan Łukasiewicz. Artistotle's Syllogistic from the standpoint of modern formal logic. Oxford: Clarendon Press, 1951.

[10] Jan Łukasiewicz. On the intuitionistic theory of deduction. Indagationes Mathematicae, 14:202212, 1952.

[11] Tadeusz Prucnal. On Two Problems of Harvey Friedman. Studia Logica, 38:247-262, 1979.

[12] Thomasz Skura. A complete syntactical characterization of the intuitionistic logic. Reports on Mathematical Logic, 23:75-80, 1989.

[13] Andrzej Wroński. Intermediate Logics and the Disjunction Property. Reports on Mathematical Logic, 1:39-51, 1973. 